\title{
IMPROVED MULTI-DIMENSIONAL MEET-IN-THE-MIDDLE CRYPTANALYSIS OF KATAN
}

\author{
Shahram Rasoolzadeh - HÅVARd RADDUM
}

\begin{abstract}
We study multidimensional meet-in-the-middle attacks on the KATAN block cipher family. Several improvements to the basic attacks are explained. The most noteworthy of these is the technique of guessing only nonlinearly involved key bits, which reduces the search space by a significant factor. The optimization decreases the complexity of multidimensional meet-in-the-middle attacks, allowing more rounds of KATAN to be efficiently attacked than previously reported.
\end{abstract}

\section{Introduction}

The KATAN family of block ciphers was designed by C a n n i èr e et al., presented at CHES 2009 [1], and has become a well-known instance of a lightweight block cipher. All versions of the KATAN family have a structure based on non-linear feedback shift registers (NLFSR). The simple round function, iterated a large number of times, allows an efficient hardware implementation and simultaneously meets the security requirements one would expect from this cipher.

KATAN has received a substantial amount of cryptanalysis since it was presented. Previous work on KATAN in the single key setting includes algebraic and cube attacks [2], conditional differential [3], differential [5], all subkeys recovery (ASR) MITM [4], 6], match-box MITM [7], multidimensional (MD)MITM [8] and dynamic cube [9]. These are all summarized in Table 1, where we also include the work of this paper.

We focus on the multidimensional (MD) meet-in-the-middle (MITM) attacks on KATAN that were introduced in [8]. The main contribution of our work is reduction in attack complexity through several optimizations. In particular, we use

(c) 2016 Mathematical Institute, Slovak Academy of Sciences.

2010 Mathematics Subject Classification: 94A60, 68P25.

Keyw ords: lightweight, block cipher, KATAN, meet-in-the-middle, reducing complexity. 
TABLE 1. Summary result of single-key attacks on KATAN family.

\begin{tabular}{|c|c|c|c|c|c|c|c|}
\hline Version & Type & Round & Time & \multicolumn{2}{|c|}{ Data } & Memory & Ref. \\
\hline \multirow{11}{*}{ KATAN 32} & Cube & 60 & $2^{39}$ & $2^{30.3}$ & $\mathrm{CP}$ & - & 2 \\
\hline & Cond. Differential & 78 & $2^{22}$ & $2^{22}$ & $\mathrm{CP}$ & - & 3 \\
\hline & Algebraic & 79 & $14.7 \mathrm{~min}$ & 20 & $\mathrm{CP}$ & - & 2 \\
\hline & ASR MITM & 110 & $2^{77}$ & 138 & KP & $2^{75.1}$ & 4 \\
\hline & Differential & 114 & $2^{77}$ & $2^{31.9}$ & KP & - & 5 \\
\hline & ASR MITM & 119 & $2^{79.1}$ & 144 & $\mathrm{CP}$ & $2^{79.1}$ & 6 \\
\hline & Matchbox MITM & 153 & $2^{78.5}$ & $2^{5}$ & $\mathrm{CP}$ & $2^{76}$ & 7 \\
\hline & Dynamic Cube & 154 & $2^{78.5}$ & $2^{32}$ & - & $2^{32}$ & 9 \\
\hline & MD MITM & 175 & $2^{78.3}$ & 3 & KP & $2^{79.6}$ & 8 \\
\hline & MD MITM & 201 & $2^{78.1}$ & 3 & KP & $2^{78.1}$ & Tab. 5 \\
\hline & MD MITM & 206 & $2^{79}$ & 3 & $\mathrm{KP}$ & $2^{78.1}$ & Tab. 5 \\
\hline \multirow{9}{*}{ KATAN 48} & Cube & 40 & $2^{49}$ & $2^{25.9}$ & $\mathrm{CP}$ & - & 2 \\
\hline & Algebraic & 64 & 6.4 hour & 5 & $\mathrm{CP}$ & - & 2 \\
\hline & Cond. Differential & 70 & $2^{34}$ & $2^{34}$ & $\mathrm{CP}$ & - & 3 \\
\hline & ASR MITM & 100 & $2^{78}$ & 128 & KP & $2^{78}$ & 4 \\
\hline & MITM ASR & 105 & $2^{79.1}$ & 144 & $\mathrm{CP}$ & $2^{79.1}$ & 6 \\
\hline & Matchbox MITM & 129 & $2^{78.5}$ & 32 & $\mathrm{CP}$ & $2^{76}$ & 7] \\
\hline & MD MITM & 130 & $2^{79.5}$ & 2 & KP & $2^{79}$ & 8 \\
\hline & MD MITM & 146 & $2^{78.1}$ & 2 & KP & $2^{77}$ & Tab. 3 \\
\hline & MD MITM & 148 & $2^{79}$ & 2 & KP & $2^{77}$ & Tab. 3 \\
\hline \multirow{9}{*}{ KATAN 64} & Cube & 30 & $2^{35}$ & $2^{20.7}$ & $\mathrm{CP}$ & - & 2 \\
\hline & Algebraic & 60 & 3.2 hour & 5 & $\mathrm{CP}$ & - & 2 \\
\hline & Cond. Differential & 68 & $2^{35}$ & $2^{35}$ & $\mathrm{CP}$ & - & 3 \\
\hline & ASR MITM & 94 & $2^{77.7}$ & 116 & KP & $2^{77.7}$ & 4 \\
\hline & ASR MITM & 99 & $2^{79.1}$ & 142 & $\mathrm{CP}$ & $2^{79.1}$ & 6 \\
\hline & MD MITM & 112 & $2^{79.5}$ & 2 & $\mathrm{KP}$ & $2^{79}$ & 8 \\
\hline & Matchbox MITM & 119 & $2^{78.5}$ & 32 & $\mathrm{CP}$ & $2^{74}$ & 7 \\
\hline & MD MITM & 126 & $2^{78.1}$ & 2 & KP & $2^{77}$ & Tab. 4 \\
\hline & MD MITM & 129 & $2^{79.0}$ & 2 & $\mathrm{KP}$ & $2^{77}$ & Tab. 4 \\
\hline
\end{tabular}

a technique of removing key bits that are not used in any non-linear operations, and show that under some mild conditions it is not necessary to guess these bits in a MITM attack.

The paper is organized as follows: Section 2 gives a brief description of KATAN, and Section 3 describes MD MITM attacks with focus on KATAN. In Section 4 we explain the optimizations we can do, and report on the improved results in Section 5 . Finally Section 6 concludes the paper. 


\section{Description of KATAN}

KATAN is a NLFSR-based family of block ciphers with block sizes of 32,48 and 64 bits. These will be referred to as KATAN 32, KATAN 48 and KATAN 64, respectively. All three versions have 254 rounds and use the same LFSR-type key schedule, accepting an 80-bit user-selected key.

The plaintext is loaded into two registers $L 1$ and $L 2$. The least significant bit (LSB) of each register, numbered 0 , is the rightmost one, and the LSB of the plaintext is loaded into the LSB of $L 2$ while its most significant bit (MSB) is loaded into the MSB of $L 1$. To update the state registers, $L 1$ and $L 2$ are shifted to the left by one bit, where the newly computed bits generated according to the following equations, are loaded into the LSBs of $L 2$ and $L 1$.

$$
\left\{\begin{array}{l}
f_{a}(L 1)=L 1\left[x_{1}\right] \oplus L 1\left[x_{2}\right] \oplus\left(L 1\left[x_{3}\right] \cdot L 1\left[x_{4}\right]\right) \oplus\left(L 1\left[x_{5}\right] \cdot I R\right) \oplus k_{a}, \\
f_{b}(L 2)=L 2\left[y_{1}\right] \oplus L 2\left[y_{2}\right] \oplus\left(L 2\left[y_{3}\right] \cdot L 2\left[y_{4}\right]\right) \oplus\left(L 2\left[y_{5}\right] \cdot L 2\left[y_{6}\right]\right) \oplus k_{b} .
\end{array}\right.
$$

Here $\oplus$ and $\cdot$ are bitwise $x o r$ and and operations, $L[x]$ denotes the $x$ th bit of $L, I R$ is a round-dependent constant, and $k_{a}$ and $k_{b}$ are two subkey bits. For round $i, 0 \leq i \leq 253, k_{a}$ and $k_{b}$ are equal to $s k_{2 i}$ and $s k_{2 i+1}$, respectively which are generated by the key schedule. Table 2 shows the parameters and tap positions associated with the different versions of KATAN. Also, the structure of KATAN 32 is shown in Fig. 1

TABle 2. Parameters of KATAN family.

\begin{tabular}{|c|ccccccccccccc|}
\hline Size & $|L 1|$ & $|L 2|$ & $x_{1}$ & $x_{2}$ & $x_{3}$ & $x_{4}$ & $x_{5}$ & $y_{1}$ & $y_{2}$ & $y_{3}$ & $y_{4}$ & $y_{5}$ & $y_{6}$ \\
\hline 32 & 13 & 19 & 12 & 7 & 8 & 5 & 3 & 18 & 7 & 12 & 10 & 8 & 3 \\
48 & 19 & 29 & 18 & 12 & 15 & 7 & 6 & 28 & 19 & 21 & 13 & 15 & 6 \\
64 & 25 & 39 & 24 & 15 & 20 & 11 & 9 & 38 & 25 & 33 & 21 & 14 & 9 \\
\hline
\end{tabular}

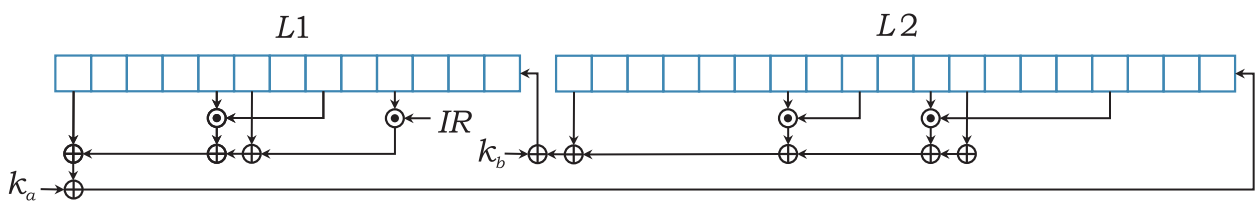

Figure 1. Structure of KATAN 32.

Applying this update procedure (i.e., shifting and loading) once is denoted as a step in this paper. One round consists of one, two or three steps with the same subkey bits, for KATAN 32, KATAN 48 and KATAN 64, respectively. After 254 rounds the content of the registers is the ciphertext. 


\section{SHAHRAM RASOOLZADEH — HÅVARD RADDUM}

The key schedule of KATAN is a linear key schedule based on an 80-bit LFSR defined by the polynomial $x_{80}+x_{61}+x_{50}+x_{13}+1$. This LFSR generates $2 \times 254=$ 508 subkey bits according to the following rule, where the $k_{i}$ 's are the userselected key bits:

$$
s k_{i}=\left\{\begin{array}{lr}
k_{i}, & 0 \leq i<80, \\
s k_{i-80} \oplus s k_{i-61} \oplus s k_{i-50} \oplus s k_{i-13}, & 80 \leq i<508 .
\end{array}\right.
$$

\section{Meet-in-the-middle attacks}

In this section we will briefly recall meet-in-the-middle attacks, and introduce the necessary notions for basic MITM and multidimensional MITM attacks. Throughout the paper, the notation $|x|$ means the bit-size of $x$.

\subsection{Basic MITM}

The basic MITM attack is a generic technique presented by Diffie and Hellman to cryptanalyze DES [10]. Despite the fact that this technique is arguably less common than differential or linear attacks on ciphers, there are some applications to specific block ciphers (such as KATAN) where using MITM principles is more successful than differential and linear attacks.

Let $E_{i, j}\left(k_{f}, S\right)$ denote the partial encryption of the cipher block $S$, beginning from the start of round $i$ and ending at the start of round $j$, where $k_{f}$ is a particular sequence of subkeys corresponding to these $j-i$ rounds. Similarly, let $D_{j, i}\left(k_{b}, S\right)$ denote the partial decryption of $S$, beginning from the start of round $j$ and ending at the start of round $i$, where $k_{b}$ is the sequence of subkeys corresponding to these $j-i$ rounds. Let $K_{f}$ and $K_{b}$ be the total set of subkey sequences that $k_{f}$ and $k_{b}$, respectively, can be drawn from.

Assume we have a cipher with $R$ rounds. The main idea of a MITM attack is that the partial keys in both parts of the cipher can be guessed separately. First, the attacker goes through all values of $k_{f}$, computes $E_{0, r}\left(k_{f}, P\right)$ for a plaintext $P$ and saves the cipher states in a table. The part of the cipher from round 0 to $r$ is called the forward side. Next, the attacker goes through all values of $k_{b}$ and computes $D_{R, r}\left(k_{b}, C\right)$ for the corresponding ciphertext. The rounds from $r$ to $R$ are called the backward side. If

$$
E_{0, r}\left(k_{f}, P\right)=D_{R, r}\left(k_{b}, C\right),
$$

then $k_{f}$ and $k_{b}$ are candidates for representing the correct secret key, and can be tested on other known plaintext/ciphertext pairs. We sum up the basic MITM attack in the following algorithm. 


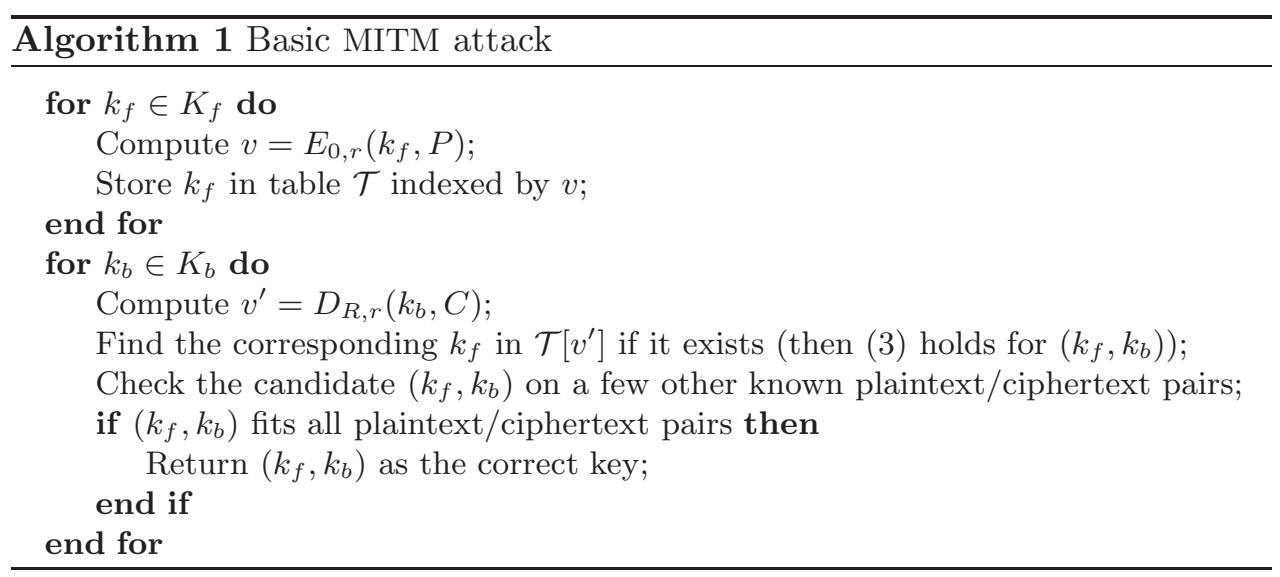

\subsection{Multidimensional MITM attack}

Multidimensional MITM attacks were first introduced in 2011 by $\mathrm{Z} \mathrm{h} \mathrm{u}$ and Go ng in [8]. In a two-dimensional (2D) MITM attack we guess a cipher block state $S$ somewhere in the middle of the cipher, and assume for the rest of the attack that the value of $S$ is known. This breaks the encryption in two parts, called dimensions in the context of MITM attacks. The idea is to combine two basic MITM attacks for each dimension, where $S$ serves as a "ciphertext" in the first dimension and a "plaintext" in the second dimension.

Note that the total complexity of a 2D MITM attack is the complexity of guessing $S$ multiplied with some part of the complexity of the two MITM attacks. To have a MD MITM attack that is faster than exhaustive key search it is therefore necessary that the key size is significantly larger than the block size. In the case of the KATAN family, the key size is 80 bits and the block sizes are 32, 48 or 64 bits.

In principle, the basic MITM attack can be extended into any number of dimensions. For a $d$-dimensional MITM attack we guess $d-1$ cipher states in the encryption procedure, dividing the cipher into $d$ dimensions. Then we perform $d$ basic MITM attacks in parallel in each dimension. To be faster than exhaustive search it is a necessary condition that $(d-1) \times|S|<|K|$. Hence we can implement 2D MITM attack to all 3 versions of KATAN and also 3D MITM attack to KATAN 32.

\subsubsection{MITM matching when $|v|<\left|K_{f}\right|$}

In the basic MITM attack the values to match are given as the value of the cipher block $v=E_{0, r}\left(k_{f}, P\right)$ at some point in the middle of the cipher. When $|v|$ is smaller than $\left|K_{f}\right|$ there will be several $k_{f}$-values giving the same cipher state $v$. 
As we will see, this is the case for KATAN. When matching from the backward side we could get a large number of $\left(k_{f}, k_{b}\right)$ candidates to test.

In the first $d-1$ dimensions of $d$-dimensional MITM attack we would prefer to have a unique $k_{f}$-value in each cell in the table built from the forward side, so we get at most one $\left(k_{f}, k_{b}\right)$ candidate to test for each $k_{b}$-value in the backward side. We can achieve this by extending $v$ to $(v, u)$, where $u$ is some value that can be computed both from $k_{f}$ and from $k_{b}$. If $|u|$ is large enough, the $(v, u)$ -value will uniquely identify $k_{f}$ in the forward side, and $k_{b}$ in the backward side. Exactly how $u$ is computed is not very important, the important thing is that $u$ is big enough to give uniqueness, and that it can be computed from both $k_{f}$ and $k_{b}$ independently.

\subsection{Implementing MD MITM on KATAN}

We will use 2D MITM and 3D MITM attacks to cryptanalyze the KATAN block ciphers. The 2D or 3D MITM attacks have the same structures, and we summarize the attacks by giving their algorithms. Algorithm 2 for 2D MITM is shown and explained below. Algorithm 3 for 3D MITM attack is similar, and given in Appendix A.

In the algorithms we use $r_{1}, \ldots, r_{5}$ as the specific rounds where either MITM matching or the guessing of a full state takes place. The numbers $r_{2}$ and $r_{4}$ denote the states that break the full cipher into dimensions, and $r_{1}, r_{3}$ and $r_{5}$ are the rounds where MITM matchings take place in the first, second and (in case of 3D) third dimensions, respectively. The value of the state separating dimensions 1 and 2 is denoted by $s$, and the value separating dimensions 2 and 3 is denoted by $t$.

The matching in the first dimension will always determine a candidate for the full key, so the matchings in the other dimensions will just work like a filter to eliminate wrong key candidates. For this reason we do not need to do matchings in the value of the full state in dimensions 2 and 3, only a few bits are needed to filter out a large fraction of key candidates from dimension 1 . The number of bits to match in the other dimension(s) is denoted by $n$.

\subsection{Complexity}

We evaluate the time and memory complexities of Algorithm 2 ,

The time complexity comes from all of the partial encryptions we need to do for all values of partial keys and all values of the guessed state in round $r_{2}$. We also do memory accesses when creating and using tables, which are not part of the encryption procedure. Also, computing $k_{f 2}$ and $k_{b 2}$ using $k_{f 1}$ and $k_{b 1}$ in the last for loop can be implemented with a matrix multiplication. These operations are few compared to the number of times we would have to iterate the shift registers generating the round keys in a naive exhaustive key search on KATAN. Since we guess the values of the keys, and we never guess more than 80 bits of the 


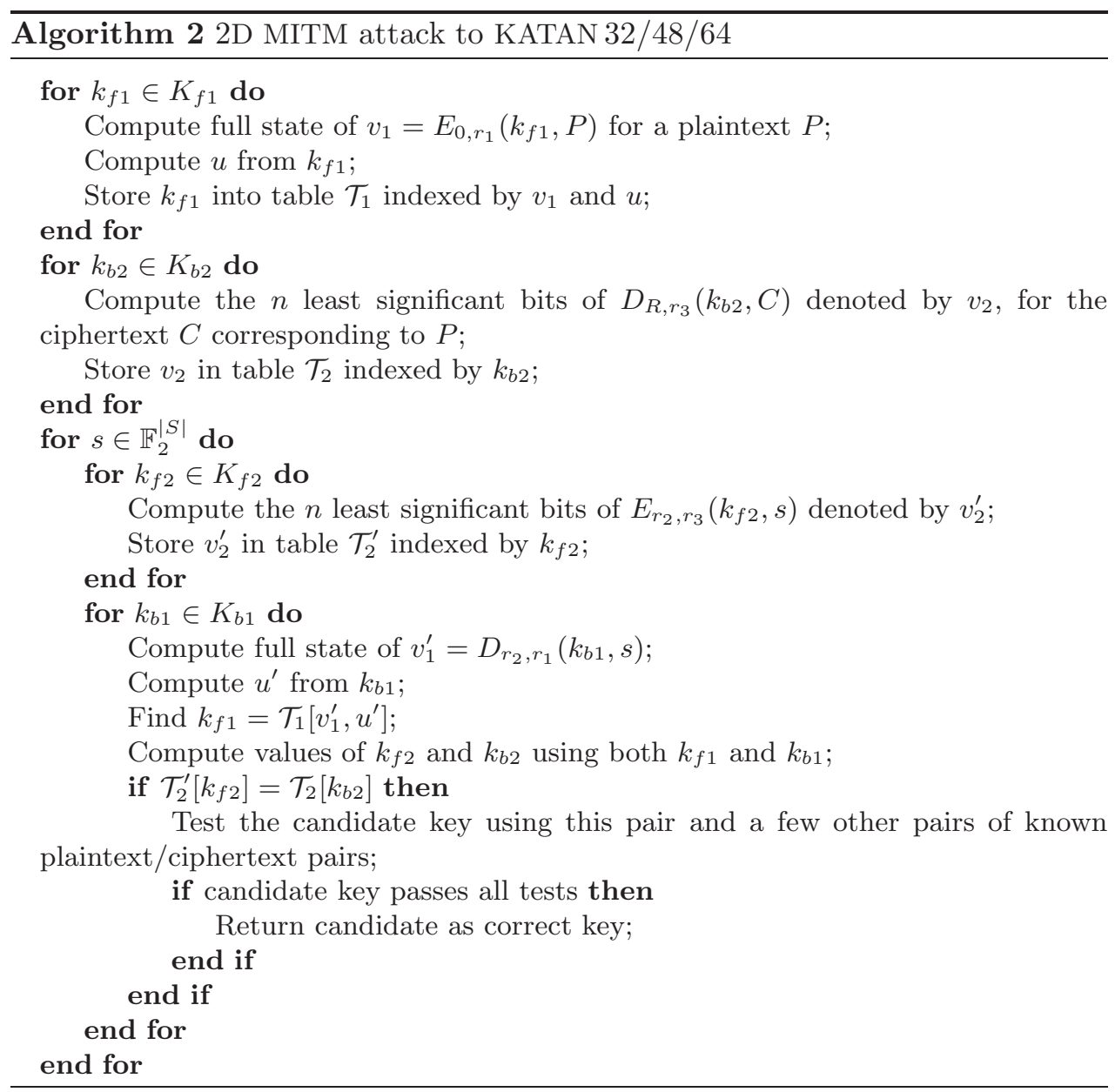

key, we do not clock the key registers in the attack. Hence we ignore the extra operations from memory access and matrix multiplication, arguing they at least cancel out with the saving from not clocking key registers that is done in a basic exhaustive search.

We give the time complexity in terms of full $R$-round encryptions. The first for-loop in Algorithm 2 iterates $2^{\left|K_{f 1}\right|}$ times, executing a $r_{1} / R$ encryption every time. Similarly, the second for-loop costs $2^{\left|K_{b 2}\right|} \times\left(R-r_{3}\right) / R$ encryptions. The complexity for the other two for-loops can be computed similarly, but these must be done once for every guess of the state $S$ in round $r_{2}$, and hence must be multiplied with $2^{|S|}$. Finally, the matching $\mathcal{T}_{2}^{\prime}\left[k_{f 2}\right]=\mathcal{T}_{2}\left[k_{b 2}\right]$ succeeds with probability $2^{-n}$, which will invoke a full trial encryption on the second pair of plaintext/ciphertext. This matching is done $2^{|s|+\left|k_{b 1}\right|}$ times, 
so the time complexity for the full 2D MITM attack can be given as

$$
\frac{r_{1}}{R} 2^{\left|K_{f 1}\right|}+\frac{R-r_{3}}{R} 2^{\left|K_{b 2}\right|}+2^{|S|}\left(\frac{r_{2}-r_{1}}{R} 2^{\left|K_{b 1}\right|}+\frac{r_{3}-r_{2}}{R} 2^{\left|K_{f 2}\right|}\right)+2^{|s|+\left|k_{b 1}\right|-n}
$$

$R$-round KATAN encryptions.

The memory complexity comes from storing the tables $\mathcal{T}_{1}, \mathcal{T}_{2}$ and $\mathcal{T}_{2}^{\prime}$. The table $\mathcal{T}_{1}$ has $2^{\left|K_{f 1}\right|}$ cells, each holding one $k_{f 1}$ key. The storage needed for $\mathcal{T}_{1}$ is then $\left|k_{f 1}\right| 2^{\left|K_{f 1}\right|}$ bits. The tables $\mathcal{T}_{2}$ and $\mathcal{T}_{2}^{\prime}$ have $2^{\left|k_{b 2}\right|}$ and $2^{\left|k_{f 2}\right|}$ cells respectively, where each cell holds $n$ bits. The total memory complexity for Algorithm 2 is then given as

$$
\left|k_{f 1}\right| 2^{\left|K_{f 1}\right|}+n\left(2^{\left|k_{f 2}\right|}+2^{\left|k_{b 2}\right|}\right) \quad \text { bits. }
$$

\section{Reducing the complexity of MITM attacks}

It is possible to apply several optimizations to the MITM attacks we have described that helps speeding them up. Some of them are rather obvious or have little impact on the total complexity, and will be only briefly mentioned in Section 4.2. However, the first optimization described in Section 4.1 can make an attack faster by a high factor. The technique is general and may be applicable to other ciphers than KATAN. It is similar to a technique used in [7].

\subsection{Only guessing non-linearly involved key bits}

During a MITM attack we need to compute $v=E_{0, r}\left(k_{f}, P\right)$, for some guess of $k_{f}$. It may happen (as in the case of KATAN) that $v$ only depends linearly on some of the bits in $k_{f}$. Let these bits of $k_{f}$ be $k_{f, l}$, and let the bits that are involved non-linearly in computing $v$ be $k_{f, n}$. By the notion " $v$ depends linearly on $k_{f, l}$ " we mean that $v$ can be written as $v=v^{\prime} \oplus l_{f}$ where $v^{\prime}$ can be computed only from $k_{f, n}$, and $l_{f}$ is a bit-string that can be computed as a linear transformation of $k_{f, l}$. Another way of looking at it is that $v^{\prime}=E_{0, r}^{\prime}\left(k_{f, n}, P\right)$, where $E^{\prime}$ is the same as $E$, except that we simply ignore, or set to 0 , the $k_{f, l}$ bits.

We can do the same from the backward side and split $k_{b}$ into disjoint subsets $k_{b, l}$ and $k_{b, n}$, such that $k_{b, l}$ is only involved linearly when computing $D_{R, r}\left(k_{b}, C\right)$. Similarly to the forward side, we can define $D_{R, r}^{\prime}\left(k_{b, n}, C\right)$ to be equal to $D$, except that we ignore the $k_{b, l}$ bits that go into the computation of $D$. Finally, if $v=D_{R, r}\left(k_{b}, C\right)$ and $v^{\prime}=D_{R, r}^{\prime}\left(k_{b, n}, C\right)$ we set $v=v^{\prime} \oplus l_{b}$ where $l_{b}$ is some linear transformation of $k_{b, l}$.

Let $l_{f}$ be given as $l_{f}=k_{f, l} M_{f}$ where $M_{f}$ is a $\left|k_{f, l}\right| \times|v|$ matrix, and similarly define $l_{b}=k_{b, l} M_{b}$. Then (3) can be written as

$$
E_{0, r}^{\prime}\left(k_{f, n}, P\right) \oplus k_{f, l} M_{f}=D_{R, r}^{\prime}\left(k_{b, n}, C\right) \oplus k_{b, l} M_{b} .
$$

Assume that the key schedule of the cipher we are investigating is linear, and that $k_{f, n}$ and $k_{b, n}$ together uniquely define the user selected key. Then it is 
possible to find two matrices $N_{f}$ and $N_{b}$ such that $k_{f, l}=\left(k_{f, n}, k_{b, n}\right) N_{f}$ and $k_{b, l}=\left(k_{f, n}, k_{b, n}\right) N_{b}$. Moreover, we can split $N_{f}$ and $N_{b}$ into their top and bottom parts $N_{* 1}$ and $N_{* 2}$ such that

$$
\left\{\begin{array}{l}
k_{f, l}=\left(k_{f, n}, k_{b, n}\right) N_{f}=k_{f, n} N_{f 1} \oplus k_{b, n} N_{f 2}, \\
k_{b, l}=\left(k_{f, n}, k_{b, n}\right) N_{b}=k_{f, n} N_{b 1} \oplus k_{b, n} N_{b 2} .
\end{array}\right.
$$

Inserting this into (44), rearranging terms and setting $A_{f}=N_{f 1} M_{f} \oplus N_{b 1} M_{b}$ and $A_{b}=N_{b 2} M_{b} \oplus N_{f 2} M_{f}$ we get

$$
E_{0, r}^{\prime}\left(k_{f, n}, P\right) \oplus k_{f, n} A_{f}=D_{R, r}^{\prime}\left(k_{b, n}, C\right) \oplus k_{b, n} A_{b} .
$$

The crucial observation is that $k_{f, l}$ and $k_{b, l}$ have disappeared from the equation used for matching, hence we do not need to guess these bits when computing matching values for round $r$. Only the bits that are involved in non-linear operations need to be guessed. Also, we have rearranged the terms such that the left hand side can be computed only from $k_{f, n}$ and the right hand side only from $k_{b, n}$.

When the assumptions given here hold, we can use one side of (6) as the values to put in the table for the MITM matchings. This technique may speed up the attack by a factor $2^{\left|k_{f, l}\right|}$ and $2^{\left|k_{b, l}\right|}$ in the forward and backward sides, allowing us to cryptanalyze more rounds of the cipher.

KATAN 48 EXAMPLE. To clarify the technique, we will give the details of MITM matching for the first dimension of a 2D MITM attack to KATAN 48. Assume that we know the whole 48-bit value of $S_{60}$, and want to do a MITM matching in all 48 bits of $S_{42}$. In the forward side, for computing $E_{0,42}^{\prime}$ from the plaintext, instead of guessing all the subkey bits going into these rounds we only need to guess the 77 non-linearly involved key bits. These bits are

$$
k_{f 1, n}=\left\{k_{0}, \ldots, k_{76}\right\} .
$$

The other key bits used in this 42-round encryption are only involved linearly, so we set $k_{f 1, l}$ to be $\quad k_{f 1, l}=\left\{k_{77}, \ldots, s k_{83}\right\}$.

The bits $s k_{80}, \ldots, s k_{83}$ can be computed from $k_{f 1, n}$, so they would not need to be guessed in any case, even if we did not use the technique described in this section. The speed-up in reduced complexity comes only from not having to guess $k_{77}, k_{78}, k_{79}$, and the complexity in the forward side will therefore be reduced by a factor $2^{3}$.

In the backward side, instead of guessing all 36 subkey bits of $\left\{s k_{84}, \ldots, s k_{119}\right\}$ for computing $D_{60,42}^{\prime}$ from $S_{60}$, we only need to guess the 32 non-linearly involved subkey bits

$$
k_{b 1, n}=\left\{s k_{86}, s k_{88}, s k_{90}, \ldots, s k_{119}\right\} .
$$

The other 4 bits only gets xored to the state bits, so

$$
k_{b 1, l}=\left\{s k_{84}, s k_{85}, s k_{87}, s k_{89}\right\} .
$$




\section{SHAHRAM RASOOLZADEH — HÅVARD RADDUM}

None of the $k_{b 1, l}$ bits can be computed from $k_{b 1, n}$, so the complexity in the backward side gets reduced by a factor $2^{4}$.

In this example, $M_{f}$ will be a $7 \times 48$ matrix and $M_{b}$ will be a $4 \times 48$ matrix. The matrices $N_{f 1}$ and $N_{f 2}$ will be of sizes $77 \times 7$ and $32 \times 7$, respectively, and $N_{b 1}$ and $N_{b 2}$ of sizes $77 \times 4$ and $32 \times 4$. We have computed these matrices and verified the correctness of the technique described in this section. The actual matrices for this example can be found in Appendix B.

\subsection{Other optimizations}

To maximize the number of rounds that can be attacked we briefly mention a few other optimizations that should be used in a MITM attack on KATAN.

First, we notice that after the plaintext has been loaded into the registers, in the first few steps the key bits are only xored onto the state and are not involved in the non-linear operations. We can therefore clock the registers up until the first key-bits go into a non-linear operation, and treat the state at this point as the (modified) plaintext. The first operation of the cipher is then to add the first key bits onto their correct cells, before clocking continues as normal. This will save us a few $f_{a}$ and $f_{b}$ steps in the beginning.

Second, if we do a MD MITM attack and only do partial matching in a dimension, some of the $f_{a}$ and $f_{b}$ operations may not affect the bits used for matching. This applies to $f_{a}$ and $f_{b}$ in the rounds close to the state used for matching. Of course, we do not need to actually compute these instances of $f_{a}$ and $f_{b}$ and so save a little in the complexity.

Third, when running through all values of $k_{f, n}$ or $k_{b, n}$ to compute matching values, we do not need to start computing from the beginning of $E^{\prime}$ or $D^{\prime}$ for each guess. Instead, for the first guess we will store the states after each round up until the matching state. For the second guess, we will only backtrack to the previous state, change the value of one of the key bits there, and recompute one round from the stored state to get a new key guess and a new matching value. In general, we will only backtrack to a state where we can make an untried key guess, and compute forward from the state stored there.

In KATAN we must guess two key bits in each round, and do st steps. If we compute $r$ rounds, and naively start from the beginning for each key guess, we must compute $4^{r} \times r \times s t$ steps to try all keys. When storing all intermediate states and only doing minimal backtracking, the expression for the number of steps we must compute to run through all keys is

$$
4(s t+4(s t+4(s t+\ldots 4(s t) \ldots),
$$

where there are $r$ brackets. This expression can be written as $4 s t \sum_{i=0}^{r-1} 4^{i}$. Using the equality $\sum_{i=0}^{r-1} x^{i}=\frac{x^{r}-1}{x-1}$ we can simplify (17) to

$$
4\left(s t+4\left(s t+4(s t+\ldots 4(s t) \ldots)=4 \times s t \times \frac{4^{r}-1}{3} \approx 4^{r} \times \frac{4}{3} \times s t .\right.\right.
$$


The speed-up of this technique compared to starting from the beginning for each guess is approximately a factor $\frac{3 r}{4}$. In addition to guessing only non-linearly involved key bits, this acceleration contributes the most in reducing the attack complexity.

All optimizations described in this section have been used to get the final complexities for the MITM attacks on KATAN given in Section 5 .

\section{Results on KATAN}

We have applied the optimization techniques from Section 4 to MD MITM attacks on KATAN. The optimizations allow us to do the attacks in [8] with a lower complexity, and to push the attacks for more rounds before meeting the exhaustive search limit. In this section we give the details of the cryptanalysis giving the best attacks on each of KATAN 32/48/64.

\subsection{D MITM on KATAN 48}

In all our 2D MITM attacks to KATAN 48, we guess $S_{60}$ to break the cipher into two dimensions. In the first dimension, $P$ and $S_{60}$ meet each other in $S_{42}$ and for this meeting we guess the 77 key bits $k_{f 1, n}=\left\{k_{0}, \ldots, k_{76}\right\}$ in the forward side and the 32 subkey bits $k_{b 1, n}=\left\{s k_{86}, s k_{88}, s k_{90}, \ldots, s k_{119}\right\}$ in the backward side. The used value for $u$ that is computable from both $k_{f 1, n}$ and $k_{b 1, n}$ is equal to

$$
\begin{gathered}
s k_{86}, s k_{88}, s k_{93}, \ldots, s k_{102}, s k_{106}, \ldots, s k_{115}, s k_{119}, s k_{90} \oplus s k_{103}, \\
s k_{90} \oplus s k_{116}, s k_{91} \oplus s k_{104}, s k_{91} \oplus s k_{117}, s k_{92} \oplus s k_{105}, s k_{92} \oplus s k_{118} .
\end{gathered}
$$

We can adjust the values of $R, r_{3}$ and $n$ to tune the complexity of the attack. The details for some of these values in the second dimension giving the best attacks are given in Table 3 , where $T$ indicates time complexity and $M$ indicates memory complexity.

\subsection{D MITM on KATAN 64}

We break the cipher into two dimensions by guessing $S_{51}$. In the first dimension, $P$ and $S_{51}$ meet each other in round 42 . For this matching we guess the 77 key bits $k_{f 1, n}=\left\{k_{0}, \ldots, k_{76}\right\}$ in the forward side and the 16 subkey bits $k_{b 1, n}=\left\{s k_{86}, \ldots, s k_{101}\right\}$ in the backward side. The used value for $u$ is equal to

$$
s k_{86}, \ldots, s k_{89}, s k_{93}, \ldots, s k_{101} \text {. }
$$

Again we can adjust the values of $R, r_{3}$ and $n$ to tune the complexity of the attack. The details for some of these values in the second dimension giving the best attacks are given in Table 4 . 

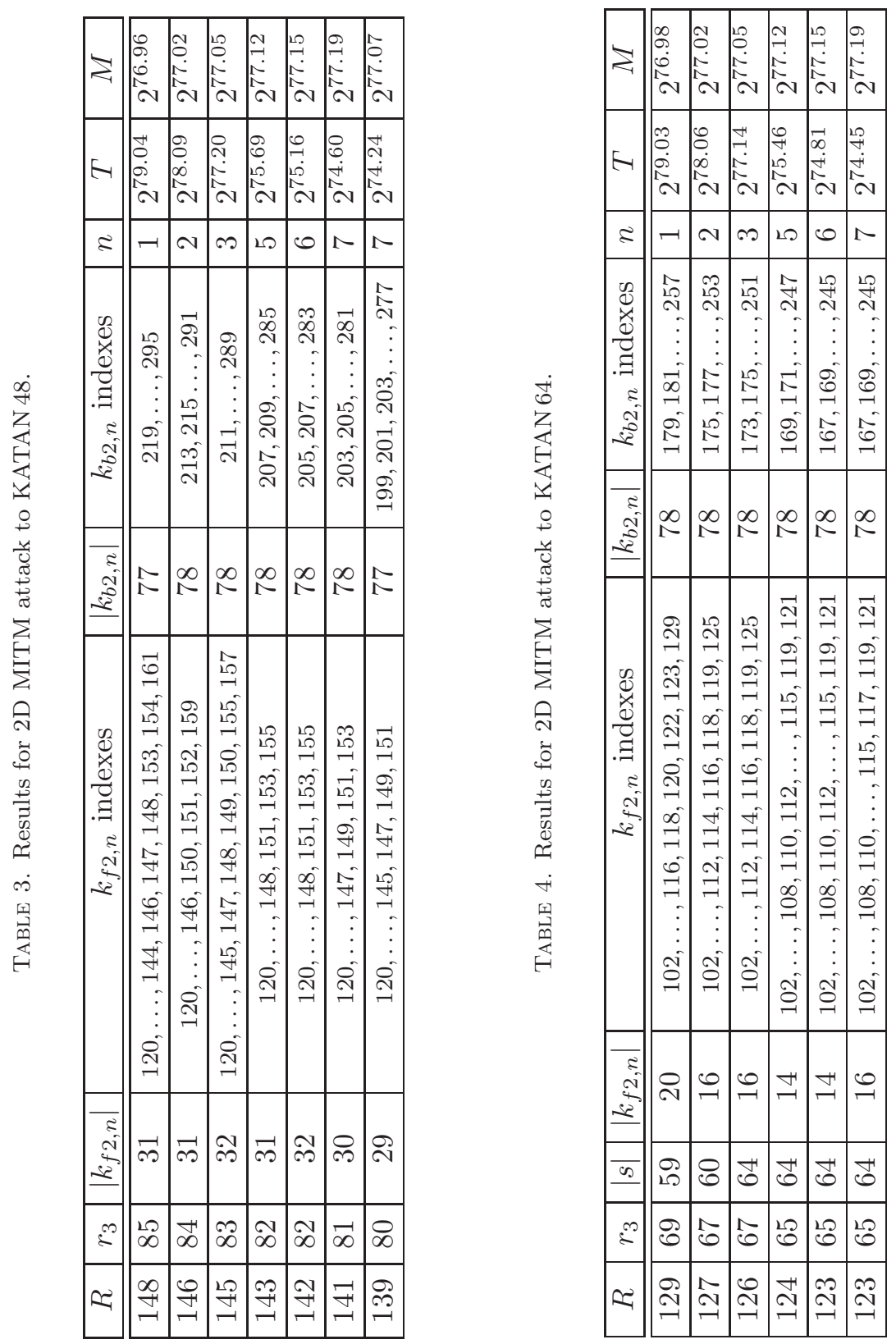
In the second dimension there is an extra twist. As all 64 bits of $S_{51}$ do not participate in the value of the $n$ matching bits in $r_{3}$, we do not need to guess all of the 64 state bits. Algorithm 2 should then be modified so only part of $s$ is guessed in the for-loop $s \in \mathbb{F}_{2}^{S}$, and the for-loop $k_{b 1} \in K_{b 1}$ is changed to also include the remaining bits of $s$. This modification allows us to guess more subkey bits in $k_{f 2, n}$.

\subsection{D MITM on KATAN 32}

For KATAN 32, we found that a 3D MITM attack gives the highest number of rounds that can be attacked. As in the 2D MITM attacks above, for the 3D MITM attacks to KATAN 32 we have the same details for full-state matching dimensions. We guess $S_{73}$ and $S_{97}$ to break the cipher into three dimensions. In the first dimension, $P$ and $S_{73}$ meet each other in $S_{44}$. For this matching we guess the 78 key bits $k_{f 1, n}=\left\{k_{0}, \ldots, k_{76}, k_{78}\right\}$ in the forward side and the 48 subkey bits $k_{b 1, n}=\left\{s k_{96}, s k_{98}, s k_{100}, \ldots, s k_{145}\right\}$ in the backward side. The used value for $u$ that is computable from both $k_{f 1, n}$ and $k_{b 1, n}$ is equal to

$$
\begin{gathered}
s k_{96}, s k_{98}, s k_{100}, s k_{101}, s k_{102}, s k_{104}, s k_{106}, \ldots, s k_{115}, s k_{117}, s k_{119}, \ldots, s k_{126}, \\
s k_{128}, s k_{130}, s k_{132}, \ldots, s k_{137}, s k_{139}, s k_{141}, s k_{143}, s k_{145}, s k_{103} \oplus s k_{116}, \\
s k_{103} \oplus s k_{127}, s k_{103} \oplus s k_{138}, s k_{103} \oplus s k_{142}, s k_{105} \oplus s k_{118}, s k_{105} \oplus s k_{131}, \\
s k_{105} \oplus s k_{140}, s k_{105} \oplus s k_{144}, s k_{103} \oplus s k_{105} \oplus s k_{129}
\end{gathered}
$$

In the second dimension, $S_{73}$ and $S_{97}$ meet each other in $S_{89}$, and for this matching we guess 32 bits $k_{f 2}=\left\{s k_{146}, \ldots, s k_{177}\right\}$ in the forward side and the 16 bits $k_{b 2}=\left\{s k_{178}, \ldots, s k_{193}\right\}$ in the backward side. Varying $R$ and $r_{5}$, the details of the attack are given in Table 5 .

\section{Conclusions}

In this work we have introduced several optimizations for doing a MD MITM attack. Applying these techniques on KATAN allows efficient attacks on more rounds than published previously, before reaching the exhaustive search bound. The tables in Section 5 give an idea of how the time and memory complexities scale when adjusting the attack parameters. The conclusion is that there exist attacks on 206-round KATAN 32, 148-round KATAN 48 and 129-round KATAN 64 that are close to the exhaustive search bound.

Estimating the exact time complexities taking into account all minor operations done in the attacks is complicated, and it is difficult to say for sure exactly when the time complexity goes above the exhaustive search bound when the number of rounds are increased. The time used for memory access to the big tables will depend on the specific implementation. However, it is clear that the optimizations presented here improve on the previous work. 


\begin{tabular}{|c|c|c|c|c|c|c|c|}
\hline$z$ & $\begin{array}{l}\stackrel{2}{0} \\
\infty \\
\stackrel{0}{\alpha} \\
\stackrel{N}{N}\end{array}$ & 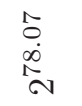 & $\begin{array}{l}\mathcal{N} \\
\infty \\
\infty \\
\stackrel{N}{N}\end{array}$ & 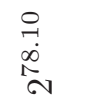 & $\left|\begin{array}{l}\mathcal{N} \\
N \\
\infty \\
\infty \\
N \\
N\end{array}\right|$ & 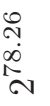 & $\begin{array}{l}\vec{\infty} \\
\infty \\
\infty \\
\infty \\
\sim\end{array}$ \\
\hline$E_{1}$ & $\begin{array}{l}\mathscr{O} \\
\dot{0} \\
\stackrel{D}{N}\end{array}$ & 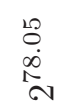 & $\begin{array}{l}\stackrel{m}{\stackrel{2}{N}} \\
\stackrel{N}{N}\end{array}$ & 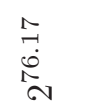 & 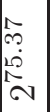 & 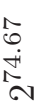 & 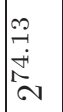 \\
\hline$\approx$ & -1 & N & $\infty$ & $\forall$ & 20 & 0 & $N$ \\
\hline 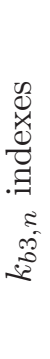 & 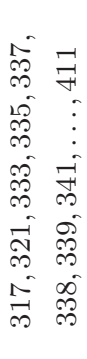 & 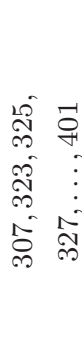 & 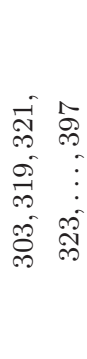 & 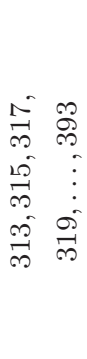 & 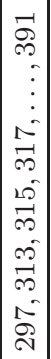 & 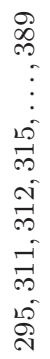 & 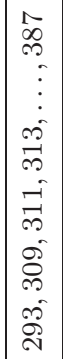 \\
\hline 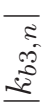 & $\infty$ & $\stackrel{\infty}{N}$ & $\stackrel{\infty}{N}$ & $\infty$ & $\infty$ & $\infty$ & $\infty$ \\
\hline 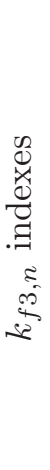 & 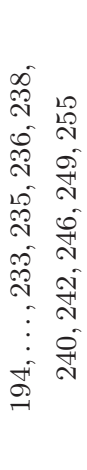 & 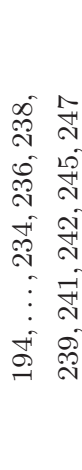 & 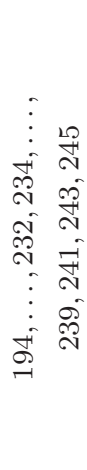 & 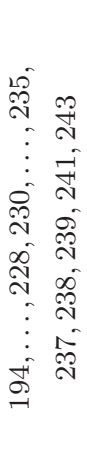 & 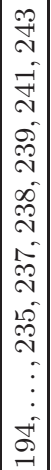 & 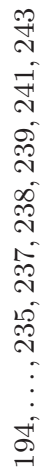 & 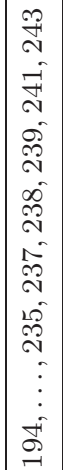 \\
\hline 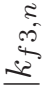 & $\stackrel{\infty}{\not \rightarrow}$ & 감 & $\underset{\forall}{\infty}$ & $\stackrel{0}{\sim}$ & $\stackrel{\sim}{7}$ & $\stackrel{\sim}{4}$ & 年 \\
\hline$\therefore$ & $\stackrel{\vec{m}}{\rightarrow}$ & $\stackrel{\circ}{\stackrel{\oplus}{\rightarrow}}$ & $\stackrel{\underset{\sim}{-}}{\sim}$ & 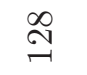 & $\left(\begin{array}{c}\infty \\
\stackrel{\sim}{-}\end{array} \mid\right.$ & 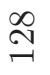 & $\stackrel{\infty}{\stackrel{N}{-1}}$ \\
\hline$\approx$ & $\underset{\sim}{\stackrel{\sim}{(})}$ & 공 & $\stackrel{\Omega}{\Omega}$ & $\stackrel{1}{\circ}$ & $\stackrel{8}{8}$ & $\stackrel{10}{2}$ & $\stackrel{\vec{\sigma}}{\sigma}$ \\
\hline
\end{tabular}




\section{REFERENCES}

[1] DE CANNIÈRE, C.-DUNKELMAN, O.-KNEŽEVIC, M.: KATAN and KTANTAN- a family of small and efficient hardware-oriented block ciphers, in: Cryptogr. Hardware and Embed. Syst.-CHES '09, The 11th Int. Workshop (C. Christophe et al., eds.) Lausanne, CH, 2009, Lecture Notes in Comput. Sci., Vol. 5747, Springer-Verlag, Berlin, 2009, pp. $272-288$.

[2] BARD, G. V.-COURTOIS, N. T.-NAKAHARA, J.-SEPEHRDAD, P.—ZHANG, B.: Algebraic, Aida/Cube and side channel analysis of KATAN family of block ciphers, in: Progress in Cryptology-INDOCRYPT'10, The 11th Int. Conf. on Cryptology, Hyderabad, India, 2010 (G. Guang et al., eds.), Lecture Notes in Comput. Sci., Vol. 6498, Springer-Verlag, Berlin, 2010, pp. 176-196.

[3] KNELLWOLF, S.-MEIER, W.-NAYA-PLASENCIA, M.: Conditional differential cryptanalysis of NLFSR-based cryptosystems, in: Adv. in Crypt.-ASIACRYPT'10, 16th Int. Conf. on the Theory and Appl. of Crypt. and Inform. Sec., Singapore (A. Masayuki, ed.), Lecture Notes in Comput. Sci., Vol. 6477, Springer-Verlag, Berlin, 2010, pp. 130-145.

[4] ISOBE, T.-SHIBUTANI, K.: All subkeys recovery attack on block ciphers: extending meet-in-the-middle approach, in: Selected Areas in Cryptography-SAC'12, 19th Int. Conf., Windsor, Canada, 2012 (L. R. Knudsen et al., eds.), Lecture Notes in Comput. Sci., Vol. 7707, Springer-Verlag, Berlin, 2012, pp. 202-221.

[5] ALBRECHT, M. R.-LEANDER, G.: An all-in-one approach to differential cryptanalysis for small block ciphers, in: Selected Areas in Cryptography-SAC'12, 19th Int. Conf., Windsor, Canada, 2012 (L. R. Knudsen et al., eds.), Lecture Notes in Comput. Sci., Vol. 7707, Springer-Verlag, Berlin, 2012, pp. 1-15.

[6] ISOBE, T.-SHIBUTANI, K.: Improved all-subkeys recovery attacks on FOX, KATAN and SHACAL-2 block ciphers, in: Int. Workshop on Fast Software Encr.-FSE'14, Lecture Notes in Comput. Sci., Vol. 8540, Springer-Verlag, Berlin, 2015, pp. 104-126.

[7] FUHR, T.-MINAUD, B.: Match box meet-in-the-middle attack against KATAN, in: Int. Workshop on Fast Software Encr.-FSE'14, Lecture Notes in Comput. Sci., Vol. 8540, Springer-Verlag, Berlin, 2015, pp. 61-81.

[8] ZHU, B.-GONG, G.: Multidimensional meet-in-the-middle attack and its applications to KATAN 32/48/64, Cryptogr. Commun. 6 (2014), 313-333.

[9] AHMADIAN, Z.-RASOOLZADEH, SH.-SAlMASIZADEH, M.-AREF, M. R.: Automated dynamic cube attack on block ciphers: cryptanalysis of SIMON and KATAN, Cryptology ePrint Archive, report 2015/040, 2015.

[10] DIFFIE, W.-HELLMAN, M.: Exhaustive cryptanalysis of the NBS data encryption standard, IEEE Comp. Soc. Press 10 (1977), 74-84.

\section{Appendix A. Algorithm for 3D MITM attack}

\section{A.1. Complexity}

The complexity of Algorithm 3 can be computed similarly to the complexity of Algorithm 2, except that we must guess two full internal states, $s$ and $t$. The time complexity can be given as

$$
\begin{aligned}
\frac{r_{1}}{R} 2^{\left|k_{f 1}\right|}+ & \frac{R-r_{5}}{R} 2^{\left|k_{b 3}\right|}+\frac{r_{5}-r_{4}}{R} 2^{|t|+\left|k_{f 3}\right|}+ \\
& 2^{|s|}\left(\frac{r_{2}-r_{1}}{R} 2^{\left|k_{b 1}\right|}+\frac{r_{3}-r_{2}}{R} 2^{\left|k_{f 2}\right|}+\frac{r_{4}-r_{3}}{R} 2^{|t|+\left|k_{b 2}\right|}\right)+2^{|s|+|t|+\left|k_{b 2}\right|-n}
\end{aligned}
$$


$R$-round encryptions of KATAN 32 .

The memory complexity for storing the tables is

$$
\left|k_{f 1}\right| 2^{\left|k_{f 1}\right|}+n\left(2^{\left|k_{b 3}\right|}+2^{|t|+\left|k_{f 3}\right|}\right)+80 \cdot 2^{\left|k_{b 1}\right|}+\left|k_{f 2}\right| 2^{\left|k_{f 2}\right|} \quad \text { bits. }
$$

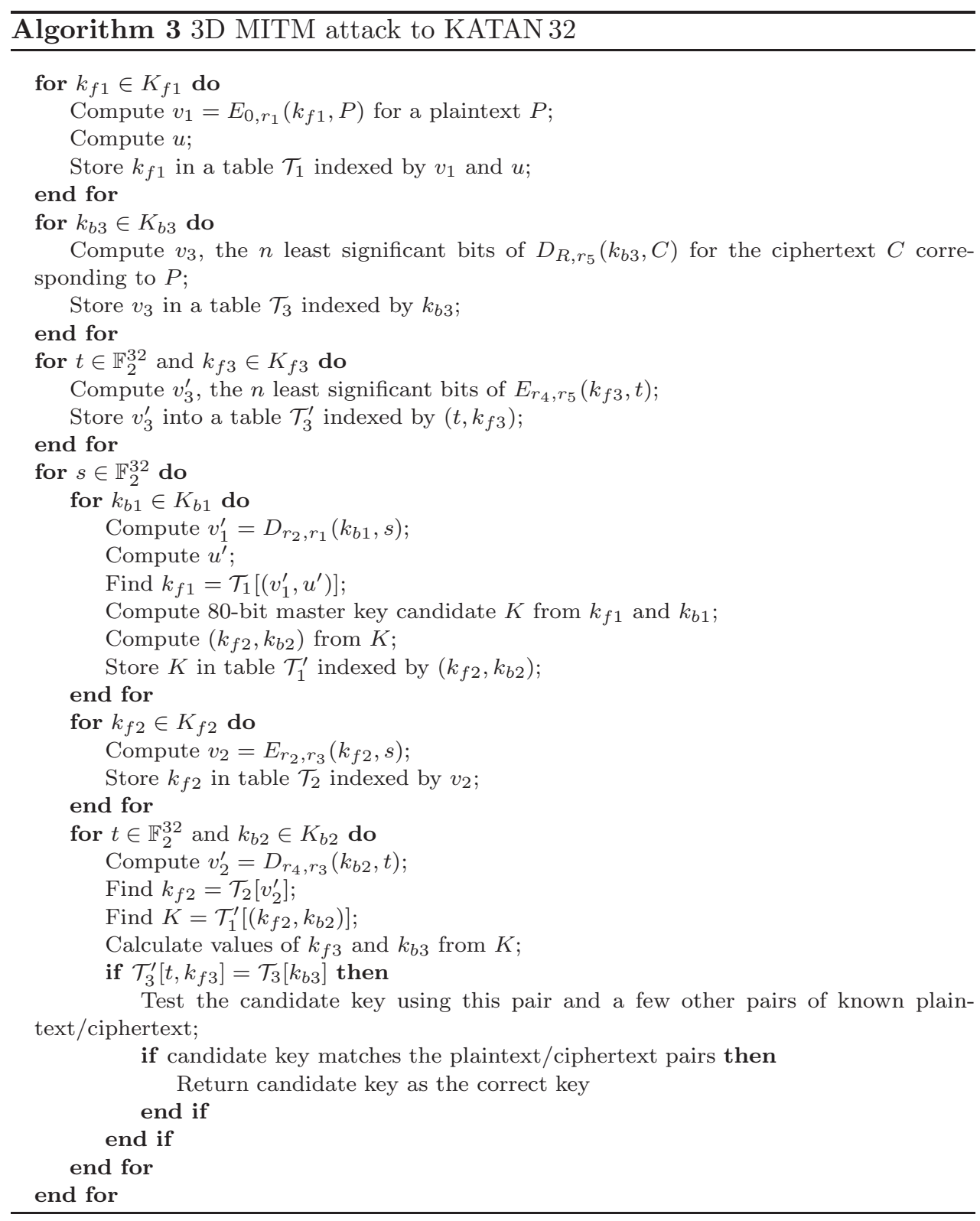




\section{Appendix B. Matrices for KATAN 48 Example}

The values for $M_{f}$ and $M_{b}$ are given below, where $O_{r \times c}$ denotes an all-zero matrix of size of $r \times c$.

$$
\begin{aligned}
& M_{f}=\left[\begin{array}{llllll:llllllllll:l}
1 & 0 & 0 & 0 & 0 & 0 & & 0 & 0 & 0 & 0 & 0 & 0 & 1 & 1 & \vdots \\
0 & 0 & 0 & 0 & 1 & 1 & & 0 & 0 & 0 & 0 & 0 & 0 & 0 & 0 & \\
0 & 0 & 0 & 0 & 0 & 0 & & 0 & 0 & 0 & 0 & 1 & 1 & 0 & 0 & \\
0 & 0 & 1 & 1 & 0 & 0 & O_{7 \times 23} & 0 & 0 & 0 & 0 & 0 & 0 & 0 & 0 & O_{7 \times 11} \\
0 & 0 & 0 & 0 & 0 & 0 & & 0 & 0 & 1 & 1 & 0 & 0 & 0 & 0 & \\
1 & 1 & 0 & 0 & 0 & 0 & & 0 & 0 & 0 & 0 & 0 & 0 & 0 & 0 & \vdots \\
0 & 0 & 0 & 0 & 0 & 0 & & 1 & 1 & 0 & 0 & 0 & 0 & 0 & 0 & \vdots
\end{array}\right],
\end{aligned}
$$

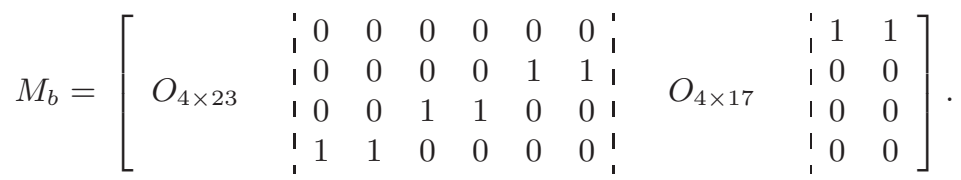

The matrices $N_{f 1}, N_{f 2}$ and $N_{b 1}$ are equal to:

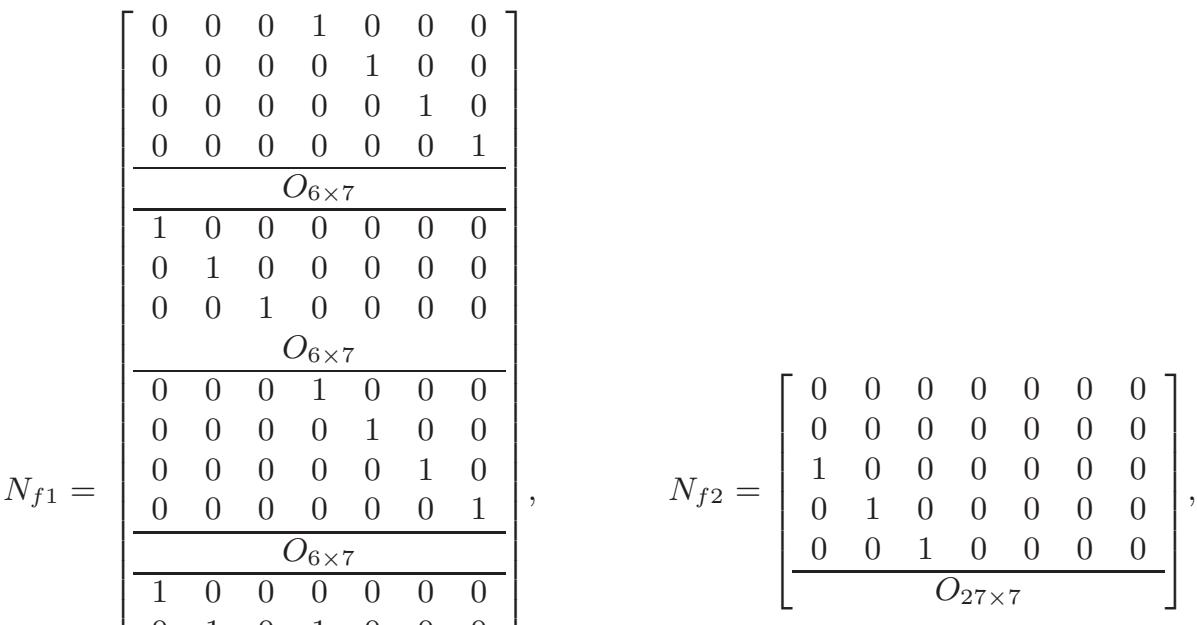

$$
\begin{aligned}
& \begin{array}{lllllll}
0 & 1 & 0 & 1 & 0 & 0 & 0
\end{array} \\
& \begin{array}{lllllll}
0 & 0 & 1 & 0 & 1 & 0 & 0
\end{array} \\
& \begin{array}{lllllll}
0 & 0 & 0 & 0 & 0 & 1 & 0
\end{array} \\
& \begin{array}{lllllll}
0 & 0 & 0 & 0 & 0 & 0 & 1
\end{array} \\
& {\left[\begin{array}{ccccccc}
\multicolumn{7}{c}{O_{6 \times 7}} \\
\hline 1 & 0 & 0 & 0 & 0 & 0 & 0 \\
0 & 1 & 0 & 0 & 0 & 0 & 0 \\
0 & 0 & 1 & 0 & 0 & 0 & 0 \\
\multicolumn{6}{c}{O_{34 \times 7}}
\end{array}\right.}
\end{aligned}
$$




\section{SHAHRAM RASOOLZADEH — HÅVARD RADDUM}

$$
N_{b 1}=\left[\begin{array}{cccc}
\multicolumn{4}{c}{O_{4 \times 4}} \\
\hline 1 & 0 & 0 & 0 \\
0 & 0 & 0 & 0 \\
0 & 1 & 0 & 0 \\
0 & 0 & 1 & 0 \\
0 & 0 & 0 & 0 \\
0 & 0 & 0 & 1 \\
\hline & O_{13 \times 4} & \\
\hline 1 & 0 & 0 & 0 \\
0 & 0 & 0 & 0 \\
0 & 1 & 0 & 0 \\
0 & 0 & 1 & 0 \\
0 & 0 & 0 & 0 \\
0 & 0 & 0 & 1 \\
\hline & O_{5 \times 4} & \\
\hline 1 & 0 & 0 & 0 \\
0 & 0 & 0 & 0 \\
0 & 1 & 0 & 0 \\
0 & 0 & 1 & 0 \\
0 & 0 & 0 & 0 \\
0 & 0 & 0 & 1 \\
\hline & O_{31} \times 4 & \\
\hline 1 & 0 & 0 & 0 \\
0 & 0 & 0 & 0 \\
0 & 1 & 0 & 0 \\
0 & 0 & 1 & 0 \\
0 & 0 & 0 & 0 \\
0 & 0 & 0 & 1
\end{array}\right],
$$

and $N_{b 2}$ is equal to $O_{32 \times 4}$.

Received August 15, 2016
Shahram Rasoolzadeh

Simula Research Laboratory

Bergen

NORWAY

Lehrstuhl Embedded Security

Ruhr-Universität Bochum

44801 Bochum

GERMANY

E-mail: shahram.Rasoolzadeh@uib.no shahram.Rasoolzadeh@ruhr-uni-bochum.de

Håvard Raddum

Simula Research Laboratory

P.O. Box 134

1325 Lysaker

NORWAY

E-mail: haavardr@simula.no 